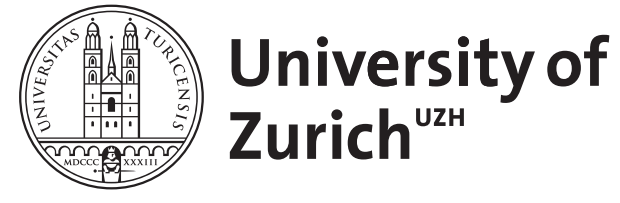
Archive

University of Zurich

University Library

Strickhofstrasse 39

CH-8057 Zurich

www.zora.uzh.ch

Year: 2003

\title{
Linear parabolic equations with singular potentials
}

\author{
Amann, $\mathrm{H}$
}

\begin{abstract}
By means of maximal regularity techniques we study the solvability of a linear heat equation with a time-dependent potential under minimal regularity assumptions.
\end{abstract}

DOI: https://doi.org/10.1007/s00028-003-0107-9

Other titles: Dedicated to Philippe Bénilan

Posted at the Zurich Open Repository and Archive, University of Zurich ZORA URL: https://doi.org/10.5167/uzh-21840

Journal Article

Accepted Version

Originally published at:

Amann, H (2003). Linear parabolic equations with singular potentials. Journal of Evolution Equations, $3(3): 395-406$.

DOI: https://doi.org/10.1007/s00028-003-0107-9 


\title{
Linear parabolic equations with singular potentials
}

\author{
HERBERT AmANn \\ In memoriam Philippe Bénilan
}

Abstract. By means of maximal regularity techniques we study the solvability of a linear heat equation with a time-dependent potential under minimal regularity assumptions.

\section{Introduction}

Let $\Omega$ be a bounded smooth domain in $\mathbb{R}^{n}$ with $n \geq 3$. Set $\Gamma:=\partial \Omega$, fix a positive number $T$, and put $J:=(0, T)$. Suppose that $a$ is a time-dependent function on $\Omega$, a 'potential', and consider the initial boundary value problem

$$
\left.\begin{array}{rlrl}
\partial_{t} u-\Delta u+a u & =0 & & \text { in } \Omega \times J, \\
u=0 & & \text { on } \Gamma \times J, \\
u(\cdot, 0) & =u^{0} & & \text { on } \Omega .
\end{array}\right\}
$$

It is the purpose of this paper to discuss the well-posedness of (1.1) under minimal regularity requirements on $a$ and $u^{0}$.

This problem has recently attracted some interest. More precisely, Brezis and Cazenave [7, Theorem A1] assume that

$$
\sigma>n / 2, \quad a \in L_{\infty}\left(J, L_{\sigma}(\Omega)\right) .
$$

Then they show that, given $p \geq 1$ and $u^{0} \in L_{p}(\Omega)$, problem (1.1) has a unique mild solution in the class

$$
C\left(\bar{J}, L_{p}(\Omega)\right) \cap L_{\infty, \text { loc }}\left(J, L_{\infty}(\Omega)\right) .
$$

Hereby a mild solution in class (1.3) is an element $u$ of (1.3) satisfying

$$
u(t)=e^{t \Delta_{D}} u^{0}+\int_{0}^{t} e^{(t-\tau) \Delta_{D}} a(\tau) u(\tau) d \tau, \quad t \in \bar{J},
$$

where $\Delta_{D}$ is the Dirichlet-Laplacian for $\Omega$.

2000 Mathematical Subject Classification: 35K20, 35K05.

Key words: Second order linear parabolic boundary value problems, generalized solutions, optimal regularity. 
They also show that uniqueness of mild solutions holds in the class

$$
L_{\infty}\left(J, L_{p}(\Omega)\right), \quad p>\sigma^{\prime}:=\sigma /(\sigma-1) .
$$

Every mild solution of (1.1) is a distributional solution, that is, an integrable function $u$ on $\Omega \times J$ satisfying

$$
\int_{J}\left\langle-\partial_{t} \varphi-\Delta \varphi+a \varphi, u\right\rangle d t=\left\langle\varphi(0), u^{0}\right\rangle
$$

for all $\varphi \in \mathcal{D}(\Omega \times[0, T))=\mathcal{D}([0, T), \mathcal{D}(\Omega))$, where $\mathcal{D}$ is the space of all smooth functions having compact support (in the indicated domain) and

$$
\langle v, w\rangle:=\int_{\Omega} v w d x
$$

In [7, Theorem A2] it is proved, in addition, that uniqueness of distributional solutions of (1.1) holds in the class (1.4), provided $p>n /(n-2)$ and

$$
a \in C\left(\bar{J}, L_{n / 2}(\Omega)\right) \text {. }
$$

This result is optimal in the sense that, given (1.6), uniqueness fails if $p=n /(n-2)$ (see [7, Remak A3]).

More recently, Hirata and Tsutsumi [10, Theorem 2.1] show that, given assumption (1.6), there exists a unique distributional solution of (1.1) in the class

$$
C\left(\bar{J}, L_{p}(\Omega)\right) \cap L_{p}\left(J, L_{p n /(n-2)}(\Omega)\right) \cap L_{\infty, \text { loc }}\left(J, L_{q}(\Omega)\right)
$$

for any $q<\infty$, provided $u^{0} \in L_{p}(\Omega)$ and $p>1$. These authors also show [10, Theorem 4.2] that there exist potentials

$$
a \in L_{\infty}\left(J, L_{n / 2}(\Omega)\right)
$$

such that uniqueness fails in the class (1.4) for $p>n /(n-2)$. Furthermore, they show (see [10, Theorem 3.1]) that there are $a$ satisfying (1.6) and $u^{0} \in L_{1}(\Omega)$ such that (1.1) has no distributional solution $u$ such that $a u$ is locally integrable on $\Omega \times J$ and $u \in C\left(\bar{J}, L_{1}(\Omega)\right)$.

In both papers the proofs of the well-posedness assertions rely on approximation arguments, a priori estimates, and properties of the heat semigroup. In this paper we use a different approach, shedding new light on this problem. Namely, we employ maximal regularity techniques and, by this way, do not only get far reaching generalizations but also significant improvements of the above results.

\section{Main results}

For $s \in \mathbb{R}$ we denote by $H_{q}^{s}:=H_{q}^{s}(\Omega), 1<q<\infty$, the Bessel potential spaces, and by $B_{p, \rho}^{s}:=B_{p, \rho}^{s}(\Omega), \quad 1 \leq p, \rho \leq \infty$, the Besov spaces. (See [14] and [15] for precise definitions and the main properties of these spaces which we use without giving further references.) 
For $q, r \in(1, \infty)$ we set

$$
\mathbf{B}_{q, r}^{s}:=\left\{u \in B_{q, r}^{s} ; \gamma u=0\right\}, \quad 1 / q<s \leq 2,
$$

where $\gamma$ denotes the trace operator for $\Gamma$, and

$$
\mathbf{B}_{q, r}^{s}:=\left\{\begin{array}{l}
B_{q, r}^{s}, \quad-2+1 / q<s<0, \\
\left(\mathbf{B}_{q^{\prime}, r^{\prime}}^{-s}\right)^{\prime}, \quad-2 \leq s \leq-2+1 / q,
\end{array}\right.
$$

the dual spaces being determined by means of the duality pairing naturally induced by $\langle\cdot, \cdot\rangle$ (and usually again denoted by the same symbol).

We suppose that

$$
\left.\begin{array}{ll}
\text { - } & n /(n-2)<q<\infty, n / 2<\sigma \leq \infty \\
\text { - } & a \in C\left(\bar{J}, L_{n / 2}\right)+L_{\infty}\left(J, L_{\sigma}\right) .
\end{array}\right\}
$$

We also suppose that

$$
\left.\begin{array}{ll}
\text { - } & 0 \leq s<2-1 / q, 1<r<\infty \\
\text { - } & \left((f, g), u^{0}\right) \in L_{r}\left(J, H_{q}^{-s} \times W_{q}^{-1 / q}(\Gamma)\right) \times \mathbf{B}_{q, r}^{-2 / r} .
\end{array}\right\}
$$

Then we consider the initial boundary value problem

$$
\begin{aligned}
\partial_{t} u-\Delta u+a u & =f & & \text { in } \Omega \times J, \\
u & =g & & \text { on } \Gamma \times J, \\
u(\cdot, 0) & =u^{0} & & \text { on } \Omega .
\end{aligned}
$$

By an $L_{r}\left(L_{q}\right)$-solution of (2.4) we mean an element $u \in L_{r}\left(J, L_{q}\right)$ satisfying

$$
\int_{J}\left\langle-\partial_{t} \varphi-\Delta \varphi+a \varphi, u\right\rangle d t=\int_{J}\left\{\langle\varphi, f\rangle-\left\langle\partial_{\nu} \varphi, g\right\rangle_{\Gamma}\right\} d t+\left\langle\varphi(0), u^{0}\right\rangle
$$

for every $\varphi \in \mathcal{D}\left([0, T), \mathcal{D}_{0}(\bar{\Omega})\right)$, where

$$
\mathcal{D}_{0}(\bar{\Omega}):=\{\varphi \in \mathcal{D}(\bar{\Omega}) ; \varphi \mid \Gamma=0\},
$$

and $\langle\cdot, \cdot\rangle_{\Gamma}$ is the duality pairing between $W_{q}^{-1 / q}(\Gamma)$ and $W_{q}^{-1 / q}(\Gamma)^{\prime}=W_{q^{\prime}}^{1 / q}(\Gamma)$, naturally induced by

$$
(v, w) \mapsto \int_{\Gamma} v w d \sigma, \quad v, w \in C(\Gamma),
$$

with $d \sigma$ denoting the volume measure of $\Gamma$. Of course, $\partial_{\nu}$ is the normal derivative with respect to the outer unit normal on $\Gamma$. Observe that $(2.5)$ is formally obtained by multiplying (2.4) with a test function $\varphi \in \mathcal{D}\left([0, T), \mathcal{D}_{0}(\bar{\Omega})\right)$, integrating over $\Omega \times J$, integrating by parts, and using Green's formula and the initial and boundary conditions. By considering test functions $\varphi$ in $\mathcal{D}(J, \mathcal{D})=\mathcal{D}(\Omega \times J)$ only it follows from (2.5) that every $L_{r}\left(L_{q}\right)$-solution of (2.4) is a distributional solution of the first equation of (2.4).

Now we can formulate the first main result of this paper, the following existence, uniqueness, and continuity theorem. 
THEOREM 2.1. Let assumption (2.2) be valid. Then, given $\left((f, g), u^{0}\right)$ satisfying (2.3), there exists a unique $L_{r}\left(L_{q}\right)$-solution, $u$, of (2.4). Furthermore,

$$
u \in C\left(\bar{J}, \mathbf{B}_{q, r}^{-2 / r}\right),
$$

and the map $\left((f, g), u^{0}\right) \mapsto u$ is linear and continuous from the space occurring in (2.3) to

$$
L_{r}\left(J, L_{q}\right) \cap C\left(\bar{J}, \mathbf{B}_{q, r}^{-2 / r}\right) .
$$

If $f, g$, and $u^{0}$ are positive then $u$ is positive as well.

COROLLARY 2.2. Suppose that $n /(n-1)<p<\infty$, that $n / 2<\sigma \leq \infty$, and that

$$
a \in C\left(\bar{J}, L_{n / 2}\right)+L_{\infty}\left(J, L_{\sigma}\right) .
$$

Put

$$
q:= \begin{cases}p m /(n-2) & \text { if } p \geq 2 \\ p n /(n-p) & \text { if } p<2 .\end{cases}
$$

Then problem (1.1) has for each $u^{0} \in L_{p}$ a unique distributional solution in the class $L_{p \vee 2}\left(J, L_{q}\right)$.

Proof. Set $r:=p \vee 2$. Then, denoting by $\hookrightarrow$ continuous embedding,

$$
L_{p} \hookrightarrow B_{p, r}^{0} \hookrightarrow B_{q, r}^{-2 / r}
$$

thanks to $1 / q=1 / p-2 / r n$. Since $-2 / r>-2+1 / q$ it follows that $B_{q, r}^{-2 / r}=\mathbf{B}_{q, r}^{-2 / r}$. Note that $p>n /(n-1)$ implies $q>n /(n-2)$. Thus Theorem 2.1 guarantees that (1.1) has a unique $L_{p \vee 2}\left(L_{q}\right)$-solution. Since the space $\mathcal{D}:=\mathcal{D}(\Omega)$ is dense in $L_{p^{\prime}}$ and in $L_{q^{\prime}}$ it is obvious that $u$ is an $L_{p \vee 2}\left(L_{q}\right)$-solution of (1.1) iff it is a distributional solution in the class $L_{p \vee 2}\left(J, L_{q}\right)$.

We denote by $\mathcal{M}:=\mathcal{M}(\Omega)$, resp. $\mathcal{M}(\Gamma)$, the space of all bounded Radon measures on $\Omega$, resp. $\Gamma$. Then we can formulate our second main result.

THEOREM 2.3. Suppose that $n / 2<\sigma \leq \infty$ and $a \in L_{\infty}\left(J, L_{\sigma}\right)$. Then problem (2.4) has for each $r \in(1,2)$ and

$$
\left((f, g), u^{0}\right) \in L_{r}(J, \mathcal{M} \times \mathcal{M}(\Gamma)) \times \mathcal{M}
$$

a unique $L_{r}\left(L_{q}\right)$-solution, for any $q \in(1, n /(n-1))$.

COROLLARY 2.4. Let the hypotheses of Theorem 2.3 be satisfied. Then (1.1) has for each $u^{0} \in \mathcal{M}$ a unique distributional solution in the class $L_{r}\left(J, L_{q}\right)$, for any $q \in(1, n /(n-1))$.

Proof. This follows from Theorem 2.3 and the density of $\mathcal{D}$ in $C_{0}=\mathcal{M}^{\prime}$.

Given the hypotheses of Theorems 2.1 and 2.3, respectively, it can be shown that the $L_{r}\left(L_{q}\right)$-solution is more regular on $J$ if $(f, g)$ has better regularity properties. For simplicity, we do not discuss these questions here. 
The proofs of these theorems are given in Section 6. In fact, a more precise result than Theorem 2.3 is proven there (see Corollary 6.1). We also refer to [3] for a detailed study of linear parabolic equations involving measures (with respect to time and space).

\section{Maximal regularity}

Given Banach spaces $E$ and $F$, we denote by $\mathcal{L}(E, F)$ the Banach space of all bounded linear maps from $E$ into $F$. Moreover, $\mathcal{L}$ is $(E, F)$ is the set of all isomorphisms in $\mathcal{L}(E, F)$. We write $[\cdot, \cdot]_{\theta}$ for the complex, and $(\cdot, \cdot)_{\theta, p}, 1 \leq p \leq \infty$, for the real interpolation functors of exponent $\theta \in(0,1)$ (cf. [2, Section I.2] for a summary of interpolation theory).

Let $E_{0}$ and $E_{1}$ be Banach spaces such that $E_{1} \stackrel{d}{\hookrightarrow} E_{0}$, that is, $E_{1}$ is continuously and densely embedded in $E_{0}$. Then we put

$$
\mathbb{W}_{r}^{1}\left(J,\left(E_{0}, E_{1}\right)\right):=L_{r}\left(J, E_{1}\right) \cap W_{r}^{1}\left(J, E_{0}\right) .
$$

It is a Banach space with the norm

$$
\|u\|_{\mathbb{W}_{r}\left(J,\left(E_{0}, E_{1}\right)\right)}:=\|u\|_{L_{r}\left(J, E_{1}\right)}+\|\partial u\|_{L_{r}\left(J, E_{0}\right)},
$$

where $\partial$ is the distributional derivative. Furthermore, setting $E_{\theta, p}:=\left(E_{0}, E_{1}\right)_{\theta, p}$ for $\theta \in(0,1)$ and $1 \leq p \leq \infty$,

$$
\mathbb{W}_{r}^{1}\left(J,\left(E_{0}, E_{1}\right)\right) \stackrel{d}{\hookrightarrow} C\left(\bar{J}, E_{1 / r^{\prime}, r}\right)
$$

(cf. [2, Theorem III.4.10.2]). Hence $\gamma_{0} u:=u(0)$, the trace of $u \in \mathbb{W}_{r}^{1}\left(E_{0}, E_{1}\right)$ at $t=0$, is well-defined. Moreover,

$$
\mathbb{W}_{r, \tau}^{1}\left(J,\left(E_{0}, E_{1}\right)\right):=\left\{u \in \mathbb{W}_{r}^{1}\left(J,\left(E_{0}, E_{1}\right)\right) ; u(\tau)=0\right\}
$$

is for each $\tau \in \bar{J}$ a closed linear subspace of $\mathbb{W}_{r}^{1}\left(J,\left(E_{0}, E_{1}\right)\right)$.

We suppose that $1<r<\infty$ and denote by $\mathcal{M R}_{r}\left(E_{1}, E_{0}\right)$ the set of all operators $A$ in $\mathcal{L}\left(E_{1}, E_{0}\right)$ such that, given any $f \in L_{r}\left(J, E_{0}\right)$, the Cauchy problem

$$
\dot{u}+A u=f(t) \quad \text { in } J, \quad u(0)=0,
$$

possesses a unique solution $u \in \mathbb{W}_{r}^{1}\left(E_{0}, E_{1}\right)$. It follows that $\mathcal{M} \mathcal{R}_{r}\left(E_{1}, E_{0}\right)$ is open in $\mathcal{L}\left(E_{1}, E_{0}\right)$. Moreover, each $A \in \mathcal{M R}_{r}\left(E_{1}, E_{0}\right)$ is the negative infinitesimal generator of a strongly continuous analytic semigroup, $\left\{e^{-t A} ; t \geq 0\right\}$ on $E_{0}$, that is, in $\mathcal{L}\left(E_{0}\right)$.

THEOREM 3.1. Suppose that $\vartheta \in(0,1)$, that $E_{\vartheta}$ is an interpolation space of exponent $\vartheta$ between $E_{0}$ and $E_{1}$, and that

$$
A \in C\left(\bar{J}, \mathcal{M R}_{r}\left(E_{1}, E_{0}\right)\right), \quad B \in L_{\infty}\left(J, \mathcal{L}\left(E_{1}, E_{\vartheta}\right)\right) .
$$

Then

$$
\left(\partial+A+B, \gamma_{0}\right) \in \mathcal{L} \text { is }\left(\mathbb{W}_{r}^{1}\left(J,\left(E_{0}, E_{1}\right)\right), L_{r}\left(J, E_{0}\right) \times E_{1 / r^{\prime}, r}\right) .
$$

For a proof of this theorem, as well as for the assertions preceding it, we refer to [5]. 


\section{Stationary operators}

Suppose that $1<q<\infty$ and put

$$
\mathbf{H}_{q}^{s}:= \begin{cases}\left\{u \in H_{q}^{s} ; \gamma u=0\right\}, & 1 / q<s \leq 2, \\ \left(\mathbf{H}_{q^{\prime}}^{-s}\right)^{\prime}, & -2 \leq s<-1+1 / q,\end{cases}
$$

where the dual spaces are determined by means of $\langle\cdot, \cdot\rangle$. It follows that

$$
\mathbf{H}_{q}^{s}=H_{q}^{s}, \quad-2+1 / q<s<-1+1 / q,
$$

and that $L_{q} \stackrel{d}{\rightarrow} \mathbf{H}_{q}^{-2}$ (cf. [1, Section 7]). We define $\mathbf{A} \in \mathcal{L}\left(L_{q}, \mathbf{H}_{q}^{-2}\right)$ by

$$
\langle v, \mathbf{A} u\rangle_{\mathbf{H}_{q}^{-2}}:=\langle-\Delta v, u\rangle, \quad(v, u) \in \mathbf{H}_{q^{\prime}}^{2} \times L_{q}
$$

where, given a Banach space $E$, we write $\langle\cdot, \cdot\rangle_{E}$ for the duality pairing $E^{\prime} \times E \rightarrow \mathbb{C}$.

PROPOSITION 4.1. $\mathbf{A} \in \mathcal{M R}_{r}\left(L_{q}, \mathbf{H}_{q}^{-2}\right)$ for $1<r<\infty$.

Proof. For Banach spaces $E_{0}$ and $E_{1}$ with $E_{1} \stackrel{d}{\hookrightarrow} E_{0}$ we write $A \in \mathcal{B I} \mathcal{P}\left(E_{1}, E_{0}\right)$, provided $A \in \mathcal{L}\left(E_{1}, E_{0}\right)$ and $-A$ generates a strongly continuous analytic semigroup on $E_{0}$, and if there exist $M, \omega \geq 0$ and $\vartheta \in(0, \pi / 2)$ such that

$$
\left\|(\omega+A)^{i t}\right\|_{\mathcal{L}\left(E_{0}\right)} \leq M e^{\vartheta|t|}, \quad t \in \mathbb{R} .
$$

It is known that $-\Delta_{D} \in \mathcal{B I} \mathcal{P}\left(\mathbf{H}_{q}^{2}, L_{q}\right)$ (cf. [12], [11], [13], [8], for example). Hence $\mathbf{A} \in \mathcal{B} \mathcal{I} \mathcal{P}\left(L_{q}, \mathbf{H}_{q}^{-2}\right)$ by [2, Proposition V.1.5.5 and Theorem V.2.1.3]. From [2, Theorem III.4.5.2] and the fact that $\mathbf{H}_{q}^{-2}$ is isomorphic to $L_{q}$ we infer that $\mathbf{H}_{q}^{-2}$ is a UMD space. Thus the assertion is a consequence of the Dore-Venni theorem [9] (see [2, Theorem III.4.10.8]).

Next we prove a simple technical lemma.

LEMMA 4.2. Suppose that $n / 2 \leq \sigma \leq \infty$ and $1<q<\infty$. Also suppose that $0 \leq \tau<1+1 / q$ and either

$$
n / \sigma+\tau \leq 2<n / q^{\prime}+\tau
$$

or

$$
n-2+\tau<n / q \leq n / \sigma^{\prime} .
$$

Then

$$
L_{\sigma} \times L_{q} \times \mathbf{H}_{q^{\prime}}^{2-\tau} \rightarrow \mathbb{C}, \quad(b, u, v) \mapsto\langle v, b u\rangle
$$

is a continuous trilinear map.

Proof. (i) Let (4.2) be satisfied. Then we deduce from Sobolev's embedding theorem that

$$
\mathbf{H}_{q^{\prime}}^{2-\tau} \hookrightarrow H_{q^{\prime}}^{2-\tau} \hookrightarrow L_{\xi}, \quad 1 / \xi=1 / q^{\prime}-(2-\tau) / n
$$


Thus, since (4.2) implies

$$
\frac{1}{\sigma}+\frac{1}{q}+\frac{1}{\xi}=\frac{1}{\sigma}+1-(2-\tau) / n \leq 1
$$

the assertion follows from Hölder's inequality.

(ii) If (4.3) is satisfied then $\mathbf{H}_{q^{\prime}}^{2-\tau} \hookrightarrow C_{0}$ thanks to $2-\tau>n / q^{\prime}$. Since (4.3) also implies $1 / \sigma+1 / q \leq 1$, Hölder's inequality implies the assertion in this case also.

Given a function $b$, we write $M_{b}$ for the multiplication operator $u \mapsto b u$. However, if no confusion seems likely, we simply denote $M_{b}$ by $b$.

COROLLARY 4.3. Let the hypotheses of Lemma 4.2 be satisfied. Then

$$
\left(b \mapsto M_{b}\right) \in \mathcal{L}\left(L_{\sigma}, \mathcal{L}\left(L_{q}, \mathbf{H}_{q}^{\tau-2}\right)\right) .
$$

It is known that

$$
\mathbf{B}_{q, r}^{2 \theta-2} \doteq\left(\mathbf{H}_{q}^{-2}, L_{q}\right)_{\theta, r}, \quad 0<\theta<1, \quad 1<q, r<\infty
$$

where $\doteq$ means 'equal except for equivalent norms', and that

$$
\mathbf{H}_{q}^{2 \theta-2} \doteq\left[\mathbf{H}_{q}^{-2}, L_{q}\right]_{\theta}, \quad 0<2 \theta<1+1 / q, \quad 1<q<\infty,
$$

(cf. [1, Theorem 7.1]).

Now we can prove the following maximal regularity result.

THEOREM 4.4. Suppose that $n / 2 \leq \sigma \leq \infty$ and $b \in L_{\sigma}$. Also suppose that $1<q<\infty$ and either

$$
2<n / q^{\prime}
$$

or

$$
n / \sigma \leq n / q^{\prime}<2
$$

Then $\mathbf{A}+b \in \mathcal{M} \mathcal{R}_{r}\left(L_{q}, \mathbf{H}_{q}^{-2}\right)$.

Proof. (i) First suppose that $\sigma>n / 2$. If (4.7) is satisfied then we can fix $\tau \in(0,1)$ such that $(4.2)$ is true. If $(4.8)$ is valid then we fix $\tau \in(0,1)$ such that (4.3) holds. Then Corollary 4.3 implies that $M_{b} \in \mathcal{L}\left(L_{q}, \mathbf{H}_{q}^{\tau-2}\right)$. Thus, identifying A and $M_{b}$ with the constant map

$$
(t \mapsto \mathbf{A}) \in C\left(\bar{J}, \mathcal{L}\left(L_{q}, \mathbf{H}_{q}^{-2}\right)\right) \quad \text { and } \quad\left(t \mapsto M_{b}\right) \in L_{\infty}\left(J, \mathcal{L}\left(L_{q}, \mathbf{H}_{q}^{\tau-2}\right)\right)
$$

respectively, the assertion follows from (4.6), Proposition 4.1, and Theorem 3.1.

(ii) Now suppose that $\sigma=n / 2$. Proposition 4.1 and the fact that $\mathcal{M} \mathcal{R}_{r}\left(L_{q}, \mathbf{H}_{q}^{-2}\right)$ is open in $\mathcal{L}\left(L_{q}, \mathbf{H}_{q}^{-2}\right)$ guarantee the existence of $\varepsilon>0$ such that

$$
\mathbf{A}+C \in \mathcal{M R}_{r}\left(L_{q}, \mathbf{H}_{q}^{-2}\right)
$$

whenever

$$
C \in \mathcal{L}\left(L_{q}, \mathbf{H}_{q}^{-2}\right), \quad\|C\| \leq \varepsilon .
$$

Choose $d \in C(\bar{\Omega})$ such that $\|b-d\|_{L_{n / 2}} \leq \varepsilon / \kappa$, where $\kappa$ is the norm of the map (4.4) for $\tau:=0$. Then it follows that $M_{b-d}$ satisfies (4.10). Consequently, (4.9) is true for 
this choice of $C$. Since $d \in L_{\infty}$, we infer from (i) that $M_{d} \in \mathcal{L}\left(L_{q}, \mathbf{H}_{q}^{\tau-2}\right)$ for some $\tau \in(0,1)$. Thus, similarly as above,

$$
\mathbf{A}+b=\left(\mathbf{A}+M_{b-d}\right)+M_{d} \in \mathcal{M} \mathcal{R}_{r}\left(L_{q}, \mathbf{H}_{q}^{-2}\right) .
$$

This proves everything.

\section{Nonautonomous problems}

Throughout this section we suppose that

$$
\text { - } 1<r<\infty, \quad n / 2<\sigma \leq \infty \text {. }
$$

Then we consider the following hypothesis:

$\left.\begin{array}{l}\text { - } \text { either } n /(n-2)<q<\infty \text { and } a \in C\left(\bar{J}, L_{n / 2}\right)+L_{\infty}\left(J, L_{\sigma}\right) \\ \text { - } \text { or } 1<q<n /(n-2) \text { and } a \in L_{\infty}\left(J, L_{\sigma}\right) \text {. }\end{array}\right\}$

THEOREM 5.1. Let assumption (5.1) be satisfied. Then

$$
\left(\partial+\mathbf{A}+a, \gamma_{0}\right) \in \mathcal{L} \operatorname{is}\left(\mathbb{W}_{r}^{1}\left(J,\left(\mathbf{H}_{q}^{-2}, L_{q}\right)\right), L_{r}\left(J, \mathbf{H}_{q}^{-2}\right) \times \mathbf{B}_{q, r}^{-2 / r}\right) .
$$

Proof. (i) Suppose that $b \in C\left(\bar{J}, L_{n / 2}\right)$. Then we deduce from Corollary 4.3 and Theorem 4.4 that

$$
\mathbf{A}+b \in C\left(\bar{J}, \mathcal{M R}_{r}\left(L_{q}, \mathbf{H}_{q}^{-2}\right)\right) .
$$

(ii) Assume that $c \in L_{\infty}\left(J, L_{\sigma}\right)$. Since $\sigma>n / 2$, part (i) of the proof of Theorem 4.4 shows that there exists $\tau \in(0,1)$ such that

$$
M_{c} \in L_{\infty}\left(J, \mathcal{L}\left(L_{q}, \mathbf{H}_{q}^{\tau-2}\right)\right) .
$$

(iii) Finally, suppose that $a \in C\left(\bar{J}, L_{n / 2}\right)+L_{\infty}\left(J, L_{\sigma}\right)$. Choose $b \in C\left(\bar{J}, L_{n / 2}\right)$ and $c \in L_{\infty}\left(J, L_{\sigma}\right)$ such that $a=b+c$. Then the theorem follows from (i), (ii), and Theorem 3.1.

PROPOSITION 5.2. Let assumption (5.1) be satisfied. Then

$$
-\partial-\Delta_{D}+a \in \mathcal{L} \text { is }\left(\mathbb{W}_{r^{\prime}, T}^{1}\left(J,\left(L_{q^{\prime}}, \mathbf{H}_{q^{\prime}}^{2}\right), L_{r^{\prime}}\left(J, L_{q^{\prime}}\right)\right) .\right.
$$

Proof. First recall that $-\Delta_{D} \in \mathcal{M} \mathcal{R}_{r^{\prime}}\left(\mathbf{H}_{q^{\prime}}^{2}, L_{q^{\prime}}\right)$. Second, if $b \in L_{n / 2}$ then the preceding proofs show that $M_{b} \in \mathcal{L}\left(H_{q^{\prime}}^{2}, L_{q^{\prime}}\right)$. Furthermore, if $b \in L_{\sigma}$ then we can find $\tau \in(0,1)$ such that $M_{b} \in \mathcal{L}\left(H_{q^{\prime}}^{2-\tau}, L_{q^{\prime}}\right)$. Thus an obvious modification of the proof of Theorem 5.1 implies that

$$
\partial-\Delta_{D}+a \in \mathcal{L} \text { is }\left(\mathbb{W}_{r^{\prime}, 0}^{1}\left(J,\left(L_{q^{\prime}}, \mathbf{H}_{q^{\prime}}^{2}\right)\right), L_{r^{\prime}}\left(J, L_{q^{\prime}}\right)\right) .
$$

Now the assertion follows by means of the transformation $t \mapsto T-t$ (cf. [2, Subsection V.2.5]).

After theses preparations we can prove the following equivalence theorem.

THEOREM 5.3. Let assumption (5.1) be satisfied and suppose that $\left(F, u^{0}\right)$ belongs to $\mathbf{H}_{q}^{-2} \times \mathbf{B}_{q}^{-2 / r}$. Then the following are equivalent: 
(i) $u \in \mathbb{W}_{r}^{1}\left(J,\left(\mathbf{H}_{q}^{-2}, L_{q}\right)\right)$ and

$$
(\partial+\mathbf{A}+a) u=F, \quad u(0)=u^{0}
$$

(ii) $u \in L_{r}\left(J, L_{q}\right)$ and

$$
\int_{J}\left\langle-\partial_{t} v-\Delta v+a v, u\right\rangle d t=\int_{J}\langle v, F\rangle d t+\left\langle v(0), u^{0}\right\rangle
$$

for all $v \in \mathcal{D}\left([0, T), \mathcal{D}_{0}(\bar{\Omega})\right)$.

Proof. It follows from Proposition 5.2 and [2, Propositions V.2.6.2 and V.2.6.3] that (i) holds iff the integral relation in (ii) is true for all $v \in \mathbb{W}_{r^{\prime}, T}^{1}\left(J,\left(L_{q^{\prime}}, \mathbf{H}_{q}^{2}\right)\right)$. Thus the assertion is a consequence of the density of $\mathcal{D}\left([0, T), \mathcal{D}_{0}(\bar{\Omega})\right)$ in the latter space (cf. [3, Lemma 8(i)]).

\section{Proof of the main theorems; generalizations}

Now it is easy to prove Theorems 2.1 and 2.3. For this we first observe that the trace theorem implies $\partial_{\nu} \in \mathcal{L}\left(\mathbf{H}_{q^{\prime}}^{2}, W_{q^{\prime}}^{1 / q}(\Gamma)\right)$. Consequently,

$$
\left(\partial_{\nu}\right)^{\prime} \in \mathcal{L}\left(W_{q}^{-1 / q}(\Gamma), \mathbf{H}_{q}^{-2}\right) .
$$

Proof. (i) Let assumptions (2.2) and (2.3) be satisfied. Put $F:=f-\left(\partial_{\nu}\right)^{\prime} g$. Then it follows from $H_{q}^{-s}=\mathbf{H}_{q}^{-s} \hookrightarrow \mathbf{H}_{q}^{-2}$ and (6.1) that $F \in L_{r}\left(J, \mathbf{H}_{q}^{-2}\right)$. In fact,

$$
\left((f, g) \mapsto f-\left(\partial_{\nu}\right)^{\prime} g\right) \in \mathcal{L}\left(L_{r}\left(J, H_{q}^{-s} \times W_{q}^{-1 / q}(\Gamma)\right), L_{r}\left(J, \mathbf{H}_{q}^{-2}\right)\right) .
$$

Now the assertions of Theorem 2.1 - except for the positivity statement — follow from Theorems 5.1 and 5.3.

The positivity assertion is obtained by an obvious approximation argument (also see [6, Section 10]).

(ii) Let the assumptions of Theorem 2.3 be satisfied and suppose that

$$
1<q<n /(n-1) .
$$

Then $1 / q>1-1 / n$ and, consequently, $W_{q^{\prime}}^{1 / q}(\Gamma) \stackrel{d}{\hookrightarrow} C(\Gamma)$. From this we deduce that

$$
\mathcal{M}(\Gamma) \hookrightarrow W_{q}^{-1 / q}(\Gamma)
$$

Furthermore, since $1>n / q^{\prime}$,

$$
\mathbf{H}_{q^{\prime}}^{2} \stackrel{d}{\hookrightarrow} \mathbf{H}_{q^{\prime}}^{1} \stackrel{d}{\hookrightarrow} C_{0}
$$

from which we infer that

$$
\mathcal{M} \hookrightarrow \mathbf{H}_{q}^{-1} \hookrightarrow \mathbf{H}_{q}^{-2}
$$

This and (6.1) imply that

$$
\left((f, g) \mapsto f-\left(\partial_{\nu}\right)^{\prime} g\right) \in \mathcal{L}\left(L_{r}(J, \mathcal{M} \times \mathcal{M}(\Gamma)), L_{r}\left(J, \mathbf{H}_{q}^{-2}\right)\right)
$$

for $1<r<\infty$. 
Recall that

$$
\mathbf{B}_{q^{\prime}, r^{\prime}}^{s} \stackrel{d}{\hookrightarrow} C_{0}, \quad s>n / q^{\prime}, \quad 1<r<\infty .
$$

Thus $\mathcal{M} \hookrightarrow \mathbf{B}_{q, r}^{-s}$ for $s>n / q^{\prime}$ and $1<r<\infty$. Consequently,

$$
\mathcal{M} \hookrightarrow \mathbf{B}_{q, r}^{-2 / r}, \quad 2 / r+n / q>n .
$$

Since $n / q>n-1$ we see that

$$
\mathcal{M} \hookrightarrow \mathbf{B}_{q, r}^{-2 / r}, \quad 1<q<n /(n-1)
$$

if $1<r<2$. Hence the assertion of Theorem 2.3 follows also from Theorems 5.1 and 5.3 .

COROLLARY 6.1. Suppose that $1<r<\infty$. Also suppose that $n / 2<\sigma \leq \infty$ and $a \in L_{\infty}\left(J, L_{\sigma}\right)$. Then problem (2.4) has for each

$$
\left((f, g), u^{0}\right) \in L_{r}(J, \mathcal{M} \times \mathcal{M}(\Gamma)) \times \mathcal{M}
$$

a unique $L_{r}\left(L_{q}\right)$-solution, $u$, provided $1<q<n /(n-1)$ and $2 / r+n / q>n$. The map

$$
L_{r}(J, \mathcal{M} \times \mathcal{M}(\Gamma)) \times \mathcal{M} \rightarrow L_{r}\left(J, L_{q}\right), \quad\left((f, g), u^{0}\right) \mapsto u
$$

is linear and continuous. Furthermore, $u \geq 0$ if $\left((f, g), u^{0}\right) \geq 0$.

For simplicity, we have restricted ourselves to the model problem (2.4). However, the following generalizations are possible.

- The operator $-\Delta$ can be replaced by a general uniformly elliptic operator of the form

$$
-\partial_{j}\left(a_{j k} \partial_{k} u\right)+a_{j} \partial_{j} u
$$

with $a_{j k}=a_{k j} \in C^{1}(\bar{\Omega})$ and $a_{j} \in L_{\infty}\left(J, C^{1}(\bar{\Omega})\right)$ for $1 \leq j, k \leq n$ (summation convention).

- Neumann or, more generally, conormal boundary conditions can be handled also.

- Instead of a single equation one can treat strongly coupled normally parabolic systems in the sense of [1].

- Higher order systems can be studied as well.

- It suffices to assume that $\Omega$ has a compact boundary.

The main problem dealing with these generalizations is the proof of maximal regularity results for nonautonomous parabolic systems in a weak setting and with minimal regularity conditions for the coefficients. For this we refer to [5]. In [4] these results are then applied to nonlinear problems in order to identify and understand 'critical exponents' and derive optimal existence results. 


\section{References}

[1] H. Amann. Nonhomogeneous linear and quasilinear elliptic and parabolic boundary value problems. In H.J. Schmeisser, H. Triebel, editors, Function Spaces, Differential Operators and Nonlinear Analysis, pages 9-126. Teubner, Stuttgart, Leipzig, 1993.

[2] H. Amann. Linear and Quasilinear Parabolic Problems, Volume I: Abstract Linear Theory. Birkhäuser, Basel, 1995.

[3] H. Amann. Linear parabolic problems involving measures. Rev. R. Acad. Cien. Serie A. Mat. (RACSAM), 95 (2001), 85-119.

[4] H. Amann. Maximal regularity and critical growth in semilinear parabolic problems, 2002. To appear.

[5] H. Amann. Maximal regularity and weak solutions of linear parabolic equations, 2002. To appear.

[6] H. Amann, P. Quittner. Semilinear parabolic equations involving measures and low-regularity data, 2002. Preprint.

[7] H. Brezis, Th. Cazenave. A nonlinear heat equation with singular initial data. J. d'Analyse Math., 68 (1996), 277-304.

[8] R. Denk, G. Dore, M. Hieber, J. Prüss, A. Venni. New thoughts on old results of R.T. Seeley, 2002. Preprint.

[9] G. Dore, A. Venni. On the closedness of the sum of two closed operators. Math. Z., 196 (1987), 189-201.

[10] D. Hirata, M. Tsutsumi. On the well-posedness of a linear heat equation with a critical singular potential. Diff. Int. Equ., 14 (2001), 1-18.

[11] J. Prüss, H. Sohr. On operators with bounded imaginary powers in Banach spaces. Math. Z., 203 (1990), 429-452.

[12] R.T. Seeley. Complex powers of an elliptic operator. In singular integrals. Proc. Symp. Pure Math., AMS, Providence, 10 (1968), 288-307.

[13] S. Sohr. Beschränkter $H_{\infty}$-Funktionalkalkül für elliptische Randwertsysteme. Doctoral dissertation, Universität GH Kassel, Germany, 1999.

[14] H. Triebel. Interpolation Theory, Function Spaces, Differential Operators. North Holland, Amsterdam, 1978.

[15] H. Triebel. Theory of Function Spaces. Birkhäuser, Basel, 1983.

Institut für Mathematik

Universität Zürich

Winterthurerstr. 190

CH-8057 Zürich

Switzerland 